\title{
Heat Transfer Designed for Bionic Surfaces with Rib Turbulators Inspired by Alopias Branchial Arch in a Simplified Gas Turbine Transition Piece
}

\author{
Haotian Guo ${ }^{1}$, Xiao Liang ${ }^{1}$, Zhenglei Yu ${ }^{2, *}$, Tao Xu ${ }^{1}$, Tianyi Zhang ${ }^{1}$, Huan Liu ${ }^{1}$ \\ and Long $\mathrm{Ma}^{1,3}$ \\ 1 School of Mechanical Science and Engineering, Jilin University, Changchun 130022, China; \\ guoht16@mails.jlu.edu.cn (H.G.); xliang0623@sina.com (X.L.); xutao@jlu.edu.cn (T.X.); \\ zhangty0323@sina.com (T.Z.); 13019202792@163.com (H.L.); kgybaobei@sohu.com (L.M.) \\ 2 Key Lab of Bionic Engineering, Ministry of Education, Jilin University, Changchun 130022, China \\ 3 College of Aviation Warfare Service, Aviation University of Air Force, Changchun 130022, China \\ * Correspondence: yuzhenglei@hotmail.com
}

Received: 26 March 2018; Accepted: 16 May 2018; Published: 19 May 2018

\begin{abstract}
The energy needed for highly efficient heat transfer has shown a continuous growth, as the energy reduction. For highly efficient power convection, gas turbine is an important device at present. But, the design of highly efficient gas turbine is limited by the temperature and the material's temperature resistance around the inlet. One part of the inlet need to be protected from burning out is transition piece. A bionic thermal surface with rib turbulators is designed according to the turbulence function of alopias' branchial arches and is evaluated for thermo-protection enhancement in a simplified gas turbine transition piece using computational fluid dynamics (CFD) simulation. With the given diameter $(\Phi=10.26 \mathrm{~mm})$ of the impinging hole, three different horizontal distances $(S)$ from impinging holes to the front of first-row rib were solved, which were $S_{1}=20 \mathrm{~mm}, S_{2}=40 \mathrm{~mm}$, and $S_{3}=60 \mathrm{~mm}$, respectively, in case 1 . But, the results revealed that $S$ is not a significant influence factor on heat transfer efficiency. The cooling coefficient increases from 0.194 to 0.198 when the distance varies from $S_{1}$ to $S_{3}$. In case 2, rib turbulator width $(W)$ and height $(H)$ have been studied in ranges from $0.5 \times \Phi$ to $1.5 \times \Phi$. All of the numerical results indicated that the best size of the rib turbulators could improve the heat transfer efficiency to $32.5 \%$, when comparing with the smooth surface. All of the comparisons will benefit the structural design of heat transfer, which could be used for solving more severe problems in thermo-protection.
\end{abstract}

Keywords: computational fluid dynamics (CFD); numerical simulation; convective heat transfer; gas turbine; simplified transition piece model; bionic thermal surface

\section{Introduction}

The energy that is needed for highly efficient transfer has shown a continuous growth, as the energy reduction. For highly efficient power convection, gas turbine is an important device at present. In order to enhance power transform efficiency, an available approach always been applied is increasing the inlet temperature in the gas turbine [1]. The research indicates that the ability of power conversion on the turbine can be improved by $10 \%$ as the inlet temperature increasing $55 \mathrm{~K}$. However, higher gas temperature will lead to larger thermal loads on the thermal surface in the transition piece, and will even threaten the working lifespan and the reliability of gas turbine hot component [2]. Therefore, the cooling technology on such a high temperature components becomes to be a critical task. 
The transition piece, which is an important component combining the combustor and turbine, is studied by scholars for heat protection. A. Gallegos Muñoz [3] investigated the geometric construction of transition piece effected on the contours of temperature and velocity in the outlet section, by using CFD. Then, structural optimization was carried out by the Genetic Algorithms (GA). The optimized results indicated that the average inlet gas temperature and the average velocity were decreased by $2.32 \%$ and about $7.73 \%$, respectively.

Wang et al. [4,5] provided the sheath with hundreds of small impingement cooling jets can enhance the convective heat transfer coefficient by the strong forced convention coolant, which was installed over the external thermal surface of the transition piece. Both experimental and computational studies were carried out. A $1 / 7$ th section of a circle was designed to simulate a part of the dump diffuser accommodating one and two half transition pieces. The experimental results showed that the non-sheathed case provided a $40 \%$ reduction in pressure losses when comparing with the sheathed case, but $35 \%$ increase in the maximum surface temperature difference and an increase of $13-22 \%$ in other surface temperature difference, based on the temperature difference of the bulk inlet and outlet temperature. The CFD results also identified that the addition of the sheath was advised.

$\mathrm{Xu}$ [6] considered the coolant hole's angle and the injection angle of coolant flow effected on the heat transfer efficiency of impinging. The CFD simulation models were employed and the results indicated that heat transfer effectiveness was improved with the rising hole's inclination and injection angle, since the thermal surface was impinged by more coolant directly. They [7], also investigated that jet flow with water drop promoted impinging cooling efficiency increasing. They concluded the mass of $3 \times 10^{-3} \mathrm{~kg} / \mathrm{s}$ droplets with diameters of 5-35 $\mu \mathrm{m}$ could enhance the $90 \%$ cooling effectiveness and reduce $122 \mathrm{~K}$ of surface temperature. For enhancing the impinging cooling efficiency, they [8] designed pin fins in the coolant chamber to increase the turbulence, and optimized the pin fin diameter and the distance. After the pin fins were brought in, the numerical reports showed that the temperature declined of $38.77 \mathrm{~K}$, when comparing to without pin fins. With the mist injecting into the cooling chamber, the area weighted average temperature got a lower value without excess pressure loss.

The transition piece in the above literature has a smooth thermal surface. However, non-smooth thermal surfaces may have higher cooling efficiency or better convective heat transfer enhancement. How to design a surface with excellent thermal protection ability remains a challenge for researchers. After billions of years of evolution, some biological structures already have excellent properties, which could provide inspiration for thermal surface designers.

Nowadays, the excellent structure of creatures in natural evolution has provided a lot of inspiration for engineering. The analysis shows that the non-smooth structural characteristics of the livings' surface morphology can change the flow. Cui et al. [9] considered four types of bionic surfaces of reducing pressure loss, which were riblet-shaped, ridge-shaped, V-shaped, and placoid-shaped, respectively. Using the Lattice Boltzmann Method (LBM), an order for drag reduction coefficient $(\eta)$ was generated, as follows: $\eta_{\text {ridge }}>\eta_{V}>\eta_{p}>\eta_{\text {rib }}$. The results suggested that the ridge-shaped structure effected on reducing significantly, and the riblet-shaped structure could strengthen turbulence flow.

Hu et al. [10] explored the heat transfer performance of coolant stream coming out for the hollow shell, which was designed by using the bionic Barchan-dune shaped (BDS) concept. They claimed that the BDS design could make the coolant stream attach to the test surface more firmly, but with more friction loss.

Referring to turbulence flow characteristic of creatures' structure, the thermal protection of the transition piece may be solved more efficiently. In this paper, inspired by alopias' gill arch, a two-chamber rectangular model with rib turbulence is designed for enhancing heat transfer efficiency. Because the study of the biomimetic thermal surface is in comparison to parameter differences, the numerical simulations that have turned out to be more available and less expensive than the contrast experiments are carried out. The CFD method is applied to investigate the flow behavior and the heat transfer of the coolant flowing in cooling chamber with rib surface. 


\section{Bionic Design}

To adapt to the marine environment, alopias need to complete respiration by absorbing the scarce oxygen in the deep water, and the excellent morphology of its gills can help oxygen exchange efficiently. The gill morphological structures of alopias supercilious is shown in the Figure 1. The branchial arch of alopias changes the flow directing of the seawater from mouth to gill filament, meanwhile more turbulence is generated. The oxygen in the flow can be contacted with the capillaries in gill filaments easily [11]. The formation of turbulence that is inspired by the gill arches can strengthen the absorption of the oxygen; it is likely that more turbulence may improve the convective heat transfer of the coolant. In order to seek the correctness of this idea, the thermal surface with ribbed turbulators will be designed in a simplified gas turbine transition piece.
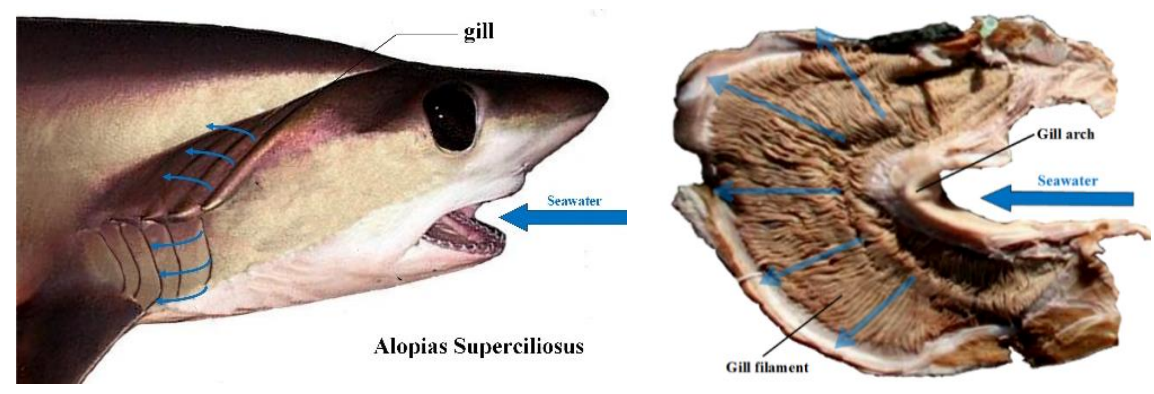

Figure 1. Gill morphological structure of Alopias [12].

Since the structure of gas turbine transition piece in operating is complex, the double-chamber simplified model is designed in this paper. In order to exclude the effect of curvature on the heat transfer, the simplified model is rectangular, as in Figure 2a. The " $\mathrm{X}$ " direction is the coolant streamwise, and the direction of the gas flow is countercurrent. The flowing length $L=1050 \mathrm{~mm}$ and the width $W=320 \mathrm{~mm}$ are used. In the figure, both sides of the below channel in the $\mathrm{X}$ axis direction are non-sealed, which is the mainstream chamber. Contrarily, one side of the above channel as the coolant chamber is opened, and the other side is closed. The height of coolant chamber and gas chamber are $38 \mathrm{~mm}$ and $162 \mathrm{~mm}$, respectively. There are three holes that are distributed in the top surface, and the diameter of all the holes $(\Phi)$ is about $10.26 \mathrm{~mm}$. The length of the rib is $320 \mathrm{~mm}$. The Figure $2 \mathrm{~b}$ shows the section of the coolant chamber with two ribs in geometry. The weight and the height of rib are set as $W$ and $H$, respectively. The distance from the first-row rib to the closed wall is $420 \mathrm{~mm}$, and the spacing of the ribs is $25 \mathrm{~mm}$. In case 1, it is considered that the streamwise distance from the holes to the front of the first-row rib is various, and is set as $20 \mathrm{~mm}, 40 \mathrm{~mm}, 60 \mathrm{~mm}$, respectively. In case 2, nine different sizes of the rectangular ribs are designed for choosing the best one for improvement of cooling efficiency.

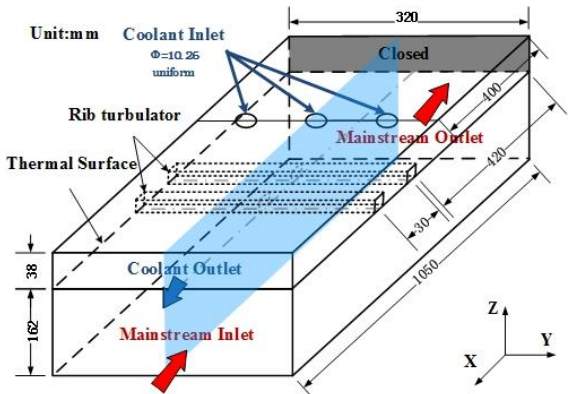

(a)

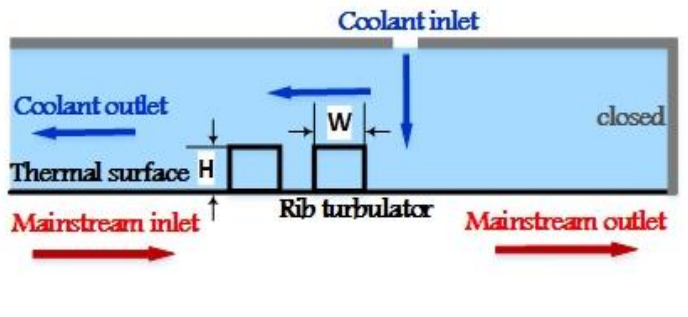

(b)

Figure 2. (a) Picture of the structure of simplified rectangular gas turbine transition piece; (b) Picture of the section of the coolant chamber with two ribs (the blue section in picture (a)). 


\section{Mathematics \& Materials}

In these cases, each hole angle with different coolant injection orientations are confirmed in the former researches, so the coolant inlet orientation is orthogonal to the wall. According to the working condition of one F model gas turbine, the temperature and the mass flux rate of the mainstream flow is set as $1300 \mathrm{~K}$ and $32.72 \mathrm{~kg} / \mathrm{s}$, respectively. The turbulence intensity is set at $5 \%$, which can be estimated based on the mass flow rate, area, hydraulic diameter of the gas inlet, and gas viscosity. In order to reduce calculation, the number of impact hole was reduced to only three in the coolant chamber. Therefore, the value of pressure on the jet hole inlet is used as the initial conditions, which is set at $1.821 \mathrm{MPa}$. The pressure recovery coefficient is given as 0.95 , which means that the ratio of pressure between the inlet and outlet of transition piece with smooth surface. The turbulent intensity on the coolant inlet is $10 \%$. Details of boundary conditions are ascertained in Table 1 [13]. In the mainstream chamber, it is assumed that the main streamflow is a mixture of $\mathrm{N}_{2}, \mathrm{O}_{2}, \mathrm{H}_{2} \mathrm{O}$, and $\mathrm{CO}_{2}$, as well as rare gases. In another chamber, air as the cooling flow is used for all of the simulations. The material of the thermal surface is using Nimonic 263 (Hucheng industry (Shanghai) Co., Ltd., Shanghai, China), for which information could be found from internet.

Table 1. Boundary conditions [13].

\begin{tabular}{ccc}
\hline Component & Boundary Conditions & Magnitude \\
\hline \multirow{3}{*}{ Mainstream inlet } & Mass flux rate & $32.72 \mathrm{~kg} / \mathrm{s}$ \\
& Gas temperature & $1300 \mathrm{~K}$ \\
& Turbulent intensity & $5 \%$ \\
& Hydraulic diameter & $0.324 \mathrm{~m}$ \\
\hline \multirow{3}{*}{ Mainstream outlet } & Pressure & $1.573 \mathrm{MPa}$ \\
& Turbulent intensity & $5 \%$ \\
& Hydraulic diameter & $0.324 \mathrm{~m}$ \\
& Convection coefficient & $10 \mathrm{~W} / \mathrm{m}^{2} \mathrm{~K}$ \\
\hline \multirow{3}{*}{ Coolant chamber } & Air temperature & $300 \mathrm{~K}$ \\
& Pressure & $1.821 \mathrm{MPa}$ \\
& Pressure recovery coefficient & 0.95 \\
& Turbulent intensity & $10 \%$ \\
& Hydraulic diameter & $0.01026 \mathrm{~m}$ \\
\hline
\end{tabular}

The model is a computational domain that is made up by hexahedron meshes in the software, ANSYS-ICEM, version 18.0. In Figure 3, the grid sensitivity test for the simplified two-chamber model with the biomimetic thermal surface are carried out. When the number of cells increase from $2,227,680$ to $2,962,080$, the area weighted average temperature is declined by $0.3 \%$. The calculation deviation of temperature is insignificant on further increasing the numbers of cells. Thus, 2,200,000 cells are used as a grid independent mesh for obtaining the solution variables in our further simulation.

In order to intuitively acquire the quantity of heat that is taken away in the process of heat exchange in the cooling chamber, the flow rate temperature $\lambda$, which is provided as an indicator that is computed by the product of flow rate and temperature, and it is defined as

$$
\lambda=\int T \rho \vec{v} \cdot d \vec{A}=\sum_{i=1}^{n} T_{i} \rho_{i} \vec{v}_{i} \cdot \vec{A}_{i}
$$

where $\rho$ is the density of the coolant and $\vec{v}$ is the facet velocity on the selected field. Also, the heat transfer coefficient can be defined as

$$
\eta=\frac{\beta_{i}-\beta_{s}}{\beta_{s}}
$$


which serves as an indicator to value the performance of impinging cooling. In Equation (2), $i$ is the number of group and $s$ represents the group of smooth thermal surface. Also, $\beta$ is defined as

$$
\beta=\lambda_{\text {out }}-\lambda_{\text {in }}
$$

In Equation (3), $\lambda_{\text {out }}$ and $\lambda_{\text {in }}$ are the flow rate temperature on the outlet and the inlet surfaces.

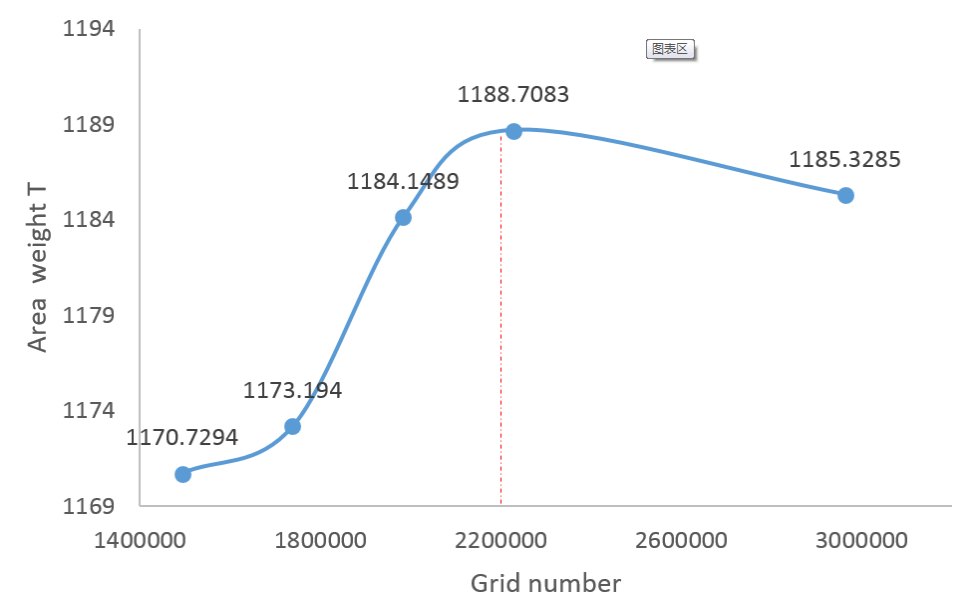

Figure 3. Validity of the grid number for air weighted average temperature.

In order to describe the contour of temperature on thermal surfaces and the distributed field of turbulent kinetic energy in the coolant chamber, this study is using the control-method, which is a commercial CFD code, ANSYS-FLUENT 18.0. The flows in these simplified calculated models are steady, Newtonian, three-dimensional, incompressible, turbulent, and behave according to three fundamental laws: continuity, and the conservation of momentum and energy. The realizable $k-\varepsilon$ turbulence model with the enhanced wall function is chosen to simulate the flow behaviors and the convective heat transfer enhancement on the biomimetic thermal surface. All of the runs were solved on a workstation with sixteen cores i7 $3.6 \mathrm{GHz}$ CPU. The decreasing of the mass residual by $85 \%$ percentage is chosen to be the standard of convergence tolerance during 2000 solving iterations.

\section{Results \& Discussion}

In this section, the contours of temperature on the thermal surface and the behaviors in the coolant chamber are present in order to explain the heat transfer efficiency and the mechanism of enhancing heat transfer. Since the pressure at the inlet of the impingement cooling holes is controllable, the effect of the rib structure on the pressure loss at the inlet and outlet will not be considered in this paper.

\subsection{Comparison of Case 1}

To investigate the influence on the streamwise distance from the cooling holes to the front of the first-row rib, contours of turbulence kinetic energy for different distances are shown as Figure 4, which presents the process of cooling impingement. The results show that the maximum turbulent kinetic energy reduced from $21,440 \mathrm{~m}^{2} / \mathrm{s}^{2}$ to $20,830 \mathrm{~m}^{2} / \mathrm{s}^{2}$, when the space increases $20 \mathrm{~mm}$ to $60 \mathrm{~mm}$ in these three groups. It can be sure that the collision of the coolant flow and the turbulent kinetic energy become weak at the front of the first-row rib, when the horizontal position of the rib is designed away from the impact cooling hole. But, the total area of turbulent kinetic energy on the thermal surface is not changed with various streamwise distance. 


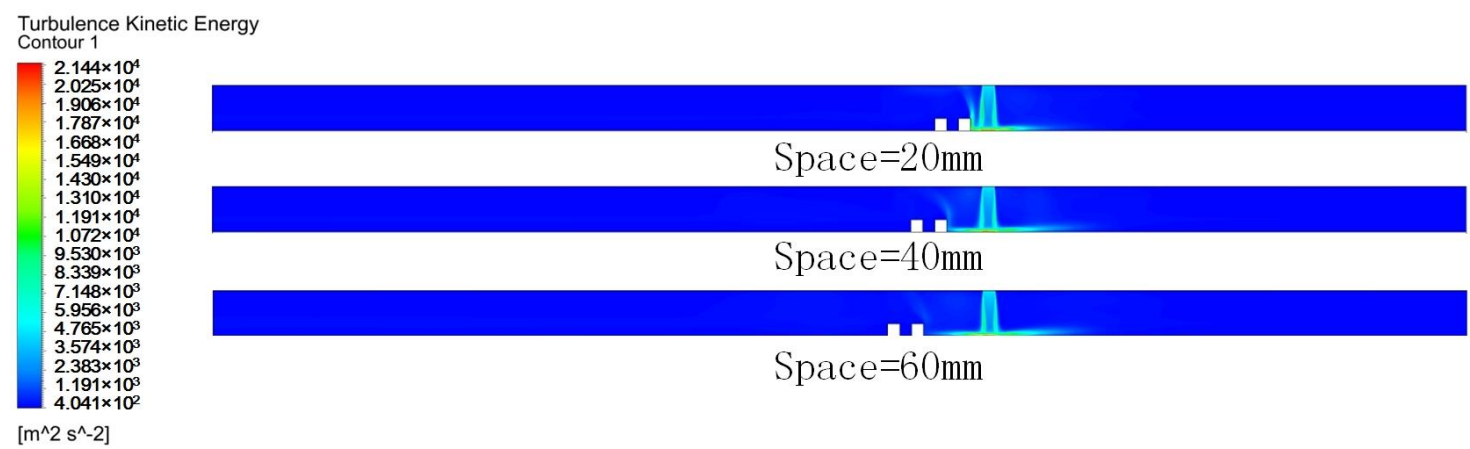

Figure 4. Contours of turbulence kinetic energy for different distances.

According to the above formats, the outlet flow rate temperature is calculated and is shown in Table 2. Due to the mass flow is conserved, the outlet flow rate temperature $\left(\lambda_{\text {out }}\right)$ can present heat taken away in the coolant chamber. In the Table 2, Negative value indicates outflow cooling chamber direction. The results show that the outlet flow rate temperature is almost the same, when the streamwise distance increases from $20 \mathrm{~mm}$ to $60 \mathrm{~mm}$. So, the quantity of heat exchanged is almost not affected by different spaces in the coolant chamber. It can be considered that the thermal protection on the transition piece has not been significantly improved. Therefore, it also can be concluded that the streamwise distance is not a significant factor affecting the heat transfer coefficient.

Table 2. Data of the outlet flow rate temperature on biomimetic thermal surface in case 1.

\begin{tabular}{ccccc}
\hline $\mathbf{N}$ & $\boldsymbol{W}(\mathbf{m m})$ & $\boldsymbol{H}(\mathbf{m m})$ & $\boldsymbol{S}(\mathbf{m m})$ & $\lambda_{\text {out }}(\mathbf{K} \cdot \mathbf{k g} / \mathbf{s})$ \\
\hline 1 & 10 & 10 & 20 & -110.3 \\
2 & 10 & 10 & 40 & -110.3 \\
3 & 10 & 10 & 60 & -110.6 \\
\hline
\end{tabular}

\subsection{Comparison of Case 2}

To investigate the effect of the sizes of rectangular ribs on the thermal surface, the width, and height of the ribs are both set as $5 \mathrm{~mm}, 10 \mathrm{~mm}$, and $15 \mathrm{~mm}$ in the case 2 . From these results, the excellent structure sizes can be found.

\subsubsection{Reflection on Temperature}

Based on the results of the numerical simulation, it can be concluded that the flow rate temperature on the coolant inlet is almost not changed as $50 \mathrm{~K} \cdot \mathrm{kg} / \mathrm{s}$. But, the outlet flow rate temperature is various with different groups, which is shown as Table 3. According to the conservation of quality, it is obviously that the larger the absolute value of $\lambda_{\text {out }}$ is, the more heat can be taken away. So, as is shown in Table 3, the simulation results indicate that the cooling efficiencies on the bionic surfaces are all clearly improved. In detail, when $W=5 \mathrm{~mm}$ and $10 \mathrm{~mm}$, the best cooling efficiency shows on $H=10 \mathrm{~mm}$. However, when $W=15 \mathrm{~mm}$, the case of $H=10 \mathrm{~mm}$ presents the worst. So, the height and width of the rib are considered that can both strongly influence the cooling performance. That is the reason why the third group has the best thermal protection effect, while the effect of the sixth group is very closed to the third. When compared with the result of the smooth thermal surface, the best cooling efficiency can be improved to $32.5 \%$.

In order to study the thermal protection on the transition piece, the comparisons of temperature distribution on thermal surfaces are shown as Figure 5. Temperature distributed on the thermal surface without rib turbulators below the injections is low, but other place is very high; however, the whole temperature that was distributed on the bionic thermal surfaces with rib turbulators is exhibited uniformly. In addition, the minimum temperature can be found on the bionic heat transfer surface and 
the data are tabulated in Table 3. The position of the lowest temperature point is mainly influenced by the height of the ribs. When $H=5 \mathrm{~mm}$, the lowest temperature appears below the impingement cooling hole, which has no difference with the smooth thermal surface. But, when $H=15 \mathrm{~mm}$, the lowest temperature is located on the upper rib facet and is closer to the hole. As shown in Table 3, the value of the lowest temperature declines with $H$ increasing. It is considered that the upper rib facet is closer to the cooling hole, with $H$ increasing and it can be rushed directly by the air from cooling holes.

Table 3. Data of flow rate temperature and cooling efficiency on thermal surface in case 2.

\begin{tabular}{cccccc}
\hline Group & $\boldsymbol{W} \mathbf{( \mathbf { m m } )}$ & $\boldsymbol{H} \mathbf{( \mathbf { m m } )}$ & $\boldsymbol{\lambda}_{\mathbf{o u t}} \mathbf{( K \cdot \mathbf { k g } / \mathbf { s } )}$ & $\boldsymbol{T}_{\min }(\mathbf{K})$ & $\boldsymbol{\eta}$ \\
\hline 1 & 0 & 0 & -83.6 & 783 & \\
2 & 5 & 5 & -105.7 & 601 & $26.4 \%$ \\
3 & 5 & 10 & -110.8 & 532 & $32.5 \%$ \\
4 & 5 & 15 & -102.0 & 467 & $22.0 \%$ \\
5 & 10 & 5 & -104.1 & 610 & $24.5 \%$ \\
6 & 10 & 10 & -110.6 & 529 & $32.3 \%$ \\
7 & 10 & 15 & -108.5 & 479 & $29.8 \%$ \\
8 & 15 & 5 & -107.4 & 593 & $28.5 \%$ \\
9 & 15 & 10 & -105.1 & 566 & $25.7 \%$ \\
10 & 15 & 15 & -108.3 & 474 & $29.5 \%$ \\
\hline
\end{tabular}

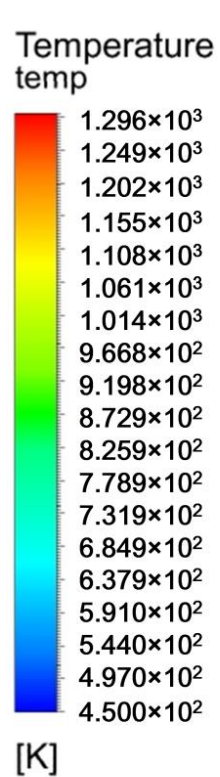

$[\mathrm{K}]$

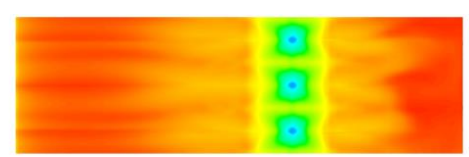

smooth

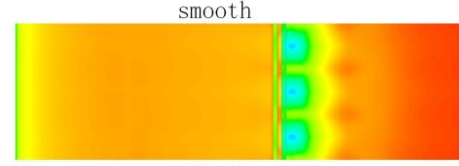

$10 \times 5$

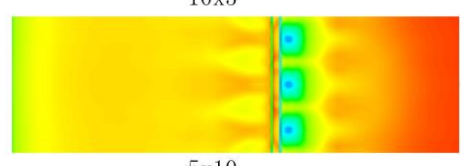

$5 \times 10$

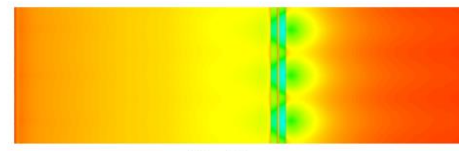

$15 \times 10$

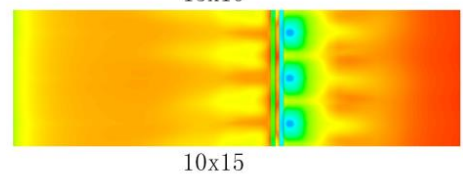

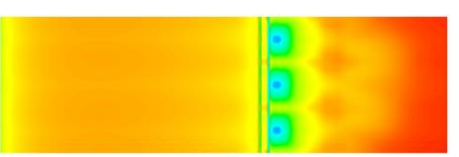

$5 \times 5$

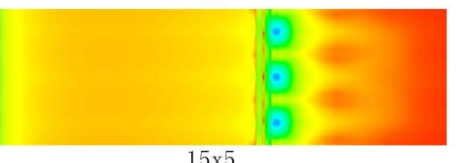

$15 \times 5$

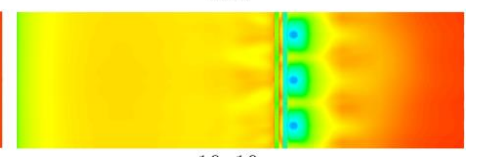

$10 \times 10$

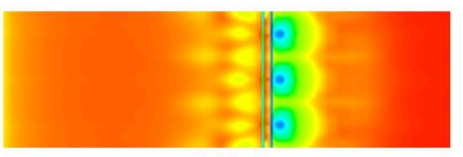

$5 \times 15$

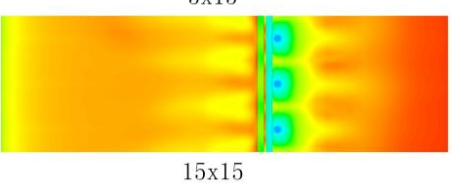

Figure 5. Comparisons of temperature distribution on the thermal surfaces.

\subsubsection{Reflection on Velocity}

To explain the mechanism of enhancing heat transfer, analyzing the flow characteristics is essential on the thermal surface in a simplified two-chamber. Figure 6 provides information on detailed flow characteristics above and nearby the rib turbulator arrangement. The result on the section in the $\mathrm{XZ}$ plane $(\mathrm{Y}=0$ in Figure 2a) indicates that the fluid vector will be altered by the convex surface, which means that the distribution of fluid has been changed. Most researches point out that the vortex makes convective heat transfer improvement on the fluid-solid surface. Two vortex can be seen in the cooling chamber of Figure 6 which were caused by inducing of double-rib structures. One is generated above the rib, with the other being located between two ribs. The height of rib $H$ is perceived to be the influence factor. When $H=5 \mathrm{~mm}$, there is amounts of space over the rib remained for vortex growing. 
However, when $H$ increased, the scale of vortex is squeezed. The larger the $H$ is, the flatter vortex become. Simultaneously, the change of $W$ can also affect the velocity and the generation of vortex, which indirectly influenced the heat transfer enhancement of convex surface. Overlarge $W$ would narrow the space between two ribs, which may bring about heat-obstruct effect as ball bearing or make vortexes hardly exist. Similarly, vortexes cannot effectively cool down the whole space if $W$ is too small. It is worth noting that oversized $H$ may cause no vortex be found between ribs. In Figure 6, the result of group $4(10 \times 15)$ shows almost no vortex during the space of double-rib and the worst result, which is opposite to all the other results with non-smooth surface.

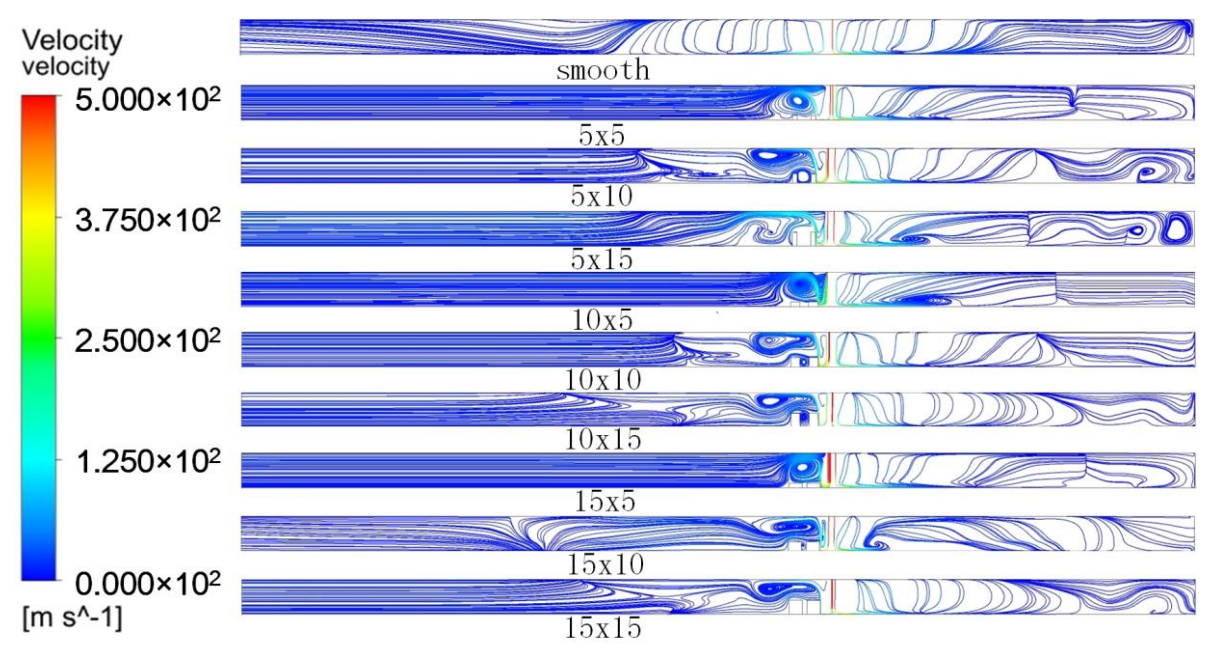

Figure 6. Cutaway views of velocity in the coolant chamber.

\subsubsection{Reflection on Turbulence Kinetic Energy}

Figure 7 shows the generation of turbulence kinetic energy in the double-chamber model in terms of contours. It is quite clear that the turbulent kinetic energy is found to be higher at the front of the first-row rib because of reflux colliding with the inlet impinging coolant. The larger $W$ leads to a closer distance from the impinging hole to the front surface of the first row that collects more kinetic energy at the corner of rib. Similar phenomenon can also be produced by increasing $H$, but it may weaken the energy spreading on streamwise. It can be observed that turbulence kinetic energy is affected less by the second-row rib, since the value of velocity from the coolant entrance is too much large and the first-row rib is too close with the coolant air column.

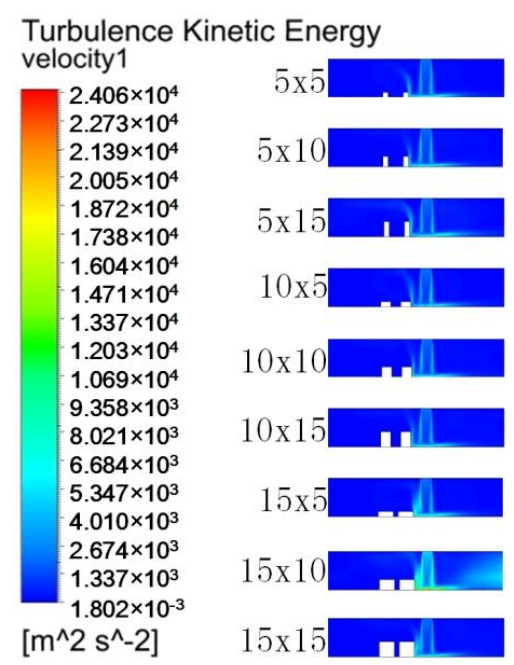

Figure 7. Contours of turbulence kinetic energy for different size rib in the coolant chamber. 


\section{Conclusions}

In this study, it has been investigated that the convective heat transfer and the coolant flow characteristics on the biomimetic thermal surface by using the realizable $k-\varepsilon$ models. The effect of rib turbulators position is discussed on the thermal surface, the variable, as well as the height and width of the rib turbulators is also studied by the comparison. The major findings of the present study are summarized below:

1. The biomimetic thermal surface inspired by Alopias' branchial arch can improve jet impingement cooling.

2. The effect of the streamwise distance from the holes to the first-row rib are studied on the biomimetic surface. It can be confirmed that the outlet flow rate temperature is almost the same, when the streamwise distance increases from $20 \mathrm{~mm}$ to $60 \mathrm{~mm}$. So, the streamwise distance is not a significant factor.

3. Since ejected into the cooling chamber at a high speed, the coolant airflow is impacted by the rib turbulators. The simulation results show that the best size of the rib turbulators can improve the heat transfer efficiency to $32.5 \%$, When comparing with the results of the smooth thermal surface.

The above conclusion and the fluid characteristics analysis provide a new sight of bionic structural design on heat transfer, especially for the gas turbine and manufacturing machines, which confront a similar severe working condition. We hope both of different researcher could make much deeper research.

Author Contributions: H.G. have completed major of this paper. X.L. have completed minor of this paper and processed the data of numerical simulation. Z.Y. and T.X. have provided the concept of this paper and guidance. T.Z., H.L. and L.M. helped me finishing the numerical tests.

Acknowledgments: The authors would like to acknowledge the National Natural Science Funds (Grant Nos. 51505181 and 51405186), the China Postdoctoral Science Foundation funded project (No. 2016M590256), the Technology Development of Jilin Province (No. 2015052106JH), the Advanced Manufacturing Project of Provincial School Construction of Jilin Province (No. SXGJSF2017-2).

Conflicts of Interest: The author declares that there is no conflict of interests regarding the publication of this paper.

\section{References}

1. Gad-Briggs, A.; Pilidis, P.; Nikolaidis, T. A Review of the Turbine Cooling Fraction for Very High Turbine Entry Temperature Helium Gas Turbine Cycles for Generation IV Reactor Power Plants. J. Nuclear Eng. Radiat. Sci. 2017, 3, 021007. [CrossRef]

2. Xue, R.; Hu, C.; Sethi, V.; Nikolaidis, T.; Pilidis, P. Effect of steam addition on gas turbine combustor design and performance. Appl. Therm. Eng. 2016, 104, 249-257. [CrossRef]

3. Munoz, A.G.; Ayala-Ramirez, V.; Alfaro-Ayala, J.A.; Acosta, B.M.T. Optimization of the transition piece applying genetic algorithms. Appl. Therm. Eng. 2011, 31, 3214-3225. [CrossRef]

4. Wang, L.; Wang, T. Investigation of the Effect of Perforated Sheath on Thermal-Flow Characteristics Over a Gas. Turbine Reverse-Flow Combustor, Part. 1-Experiment. In Proceedings of the 2013 ASME Turbo Expo: Turbine Technical Conference and Exposition, San Antonio, TX, USA, 3-7 June 2013.

5. Wang, L.; Wang, T. Investigation of the Effect of Perforated Sheath on Thermal-Flow Characteristics Over a Gas. Turbine Reverse-Flow Combustor, Part. 2-Computational Analysis. In Proceedings of the 2013 ASME Turbo Expo: Turbine Technical Conference and Exposition, San Antonio, TX, USA, 3-7 June 2013.

6. Yu, Z.L.; Xu, T.; Li, J.L.; Ma, L.; Xu, T.S. Comparison of a series of double chamber model with various hole angles for enhancing cooling effectiveness. Int. Commun. Heat Mass Transf. 2013, 44, 38-44. [CrossRef]

7. Yu, Z.L.; Xu, T.; Li, J.L.; Xu, T.S.; Yoshino, T. Computational Analysis of Droplet Mass and Size Effect on Mist/Air Impingement Cooling Performance. Adv. Mech. Eng. 2013, 5, 181856. [CrossRef]

8. Xu, T.; Xiu, H.; Li, J.L.; Ge, H.C.; Shao, Q.; Yang, G.; Yu, Z.L. Simulation of Impinging Cooling Performance with Pin Fins and Mist Cooling Adopted in a Simplified Gas. Turbine Transition Piece. Adv. Mech. Eng. 2014, 2014, 327590. [CrossRef] 
9. Cui, J.; Fu, Y.B. A Numerical Study on Pressure Drop in Microchannel Flow with Different Bionic Micro-Grooved Surfaces. J. Bionic Eng. 2012, 9, 99-109. [CrossRef]

10. Zhou, W.; Hu, H. A novel sand-dune-inspired design for improved film cooling performance. Int. J. Heat Mass Transf. 2017, 110, 908-920. [CrossRef]

11. Bernal, D.; Sepulveda, C.; Mathieu-Costello, O.; Graham, J.B. Comparative studies of high performance swimming in sharks I. Red muscle morphometrics, vascularization and ultrastructure. J. Exp. Biol. 2003, 206, 2831-2843. [CrossRef] [PubMed]

12. Wootton, T.P.; Sepulveda, C.A.; Wegner, N.C. Gill Morphometrics of the Thresher Sharks (Genus Alopias): Correlation of Gill Dimensions with Aerobic Demand and Environmental Oxygen. J. Morphol. 2015, 276, 589-600. [CrossRef] [PubMed]

13. Arturo, A.A.J.; Armando, G.M.; Manuel, R.A.J.; Polo, F.L.M.; Alfonso, C.A.; Alejandro, M.G. Analysis of the Flow in the Combustor-Transition Piece Considering the Variation in the Fuel Composition. In Proceedings of the ASME International Heat Transfer Conference, Washington, DC, USA, 8-13 August 2010; Volume 3, pp. 245-257.

(C) 2018 by the authors. Licensee MDPI, Basel, Switzerland. This article is an open access article distributed under the terms and conditions of the Creative Commons Attribution (CC BY) license (http://creativecommons.org/licenses/by/4.0/). 